\title{
Cancer-related Cognitive Impairment in Patients With Newly Diagnosed Aggressive Lymphoma Undergoing Standard Chemotherapy: a Longitudinal Feasibility Study
}

Priscilla Gates ( $\nabla$ priscilla.gates@austin.org.au )

Austin Health https://orcid.org/0000-0002-7978-5802

\section{Meinir Krishnasamy}

Peter Mac: Peter MacCallum Cancer Centre

\section{Carlene Wilson}

Austin Health

Eliza A Hawkes

Austin Health

Vincent Dore

Austin Health

Yuliya Perchyonok

Austin Health

Christopher C Rowe

Austin Health

\section{Adam K Walker}

University of New South Wales

\section{Janette L Vardy}

Concord Hospital: Concord Repatriation General Hospital

Michiel de Ruiter

Netherlands Cancer Institute: Antoni van Leeuwenhoek Nederlands Kanker Instituut

Tania Cushion

Austin Health

Haryana M Dhillon

The University of Sydney

Karla Gough

Peter MacCallum Cancer Centre

\section{Research Article}

Keywords: feasibility study, aggressive lymphoma, cancer-related cognitive impairment

Posted Date: January 3rd, 2022

DOI: https://doi.org/10.21203/rs.3.rs-1112368/v1

License: (c) (i) This work is licensed under a Creative Commons Attribution 4.0 International License. Read Full License 


\section{Abstract}

\section{Purpose}

Cancer-related cognitive impairment $(\mathrm{CRCl})$ is a recognised adverse consequence of cancer and its treatment. This study assessed the feasibility of collecting longitudinal data on cognition in patients with newly diagnosed, aggressive lymphoma undergoing standard therapy with curative intent via self-report, neuropsychological assessment, peripheral markers of inflammation and neuroimaging. An exploration and description of patterns of cancer-related cognitive impairment over the course of treatment and recovery was also explored.

\section{Methods}

Eligible participants completed repeated measures of cognition including self-report, neuropsychological assessment, blood cell-based inflammatory markers, and neuroimaging at three pre-specified time-points, Time 1 (T1) - pre-treatment (treatment naïve), Time 2 (T2) - mid-treatment, and Time 3 (T3) - six to eight weeks post-completion of treatment.

\section{Results}

Of 33 eligible participants, 30 (91\%, 95\% Cl: 76\%, 97\%) were recruited over 10 months. The recruitment rate was 3 patients/month (95\% Cl: 2.0, 4.3 patients/month). Reasons for declining included feeling overwhelmed and rapid treatment commencement. Mean age was 57 years ( $S D=17$ years) and 16/30 (53\%) were male. Most patients (20/30,67\%) had diffuse large B cell lymphoma or Hodgkin lymphoma (4/30,13\%). The neuroimaging sub-study was optional, 11/30 participants (37\%) were eligible to take part, and all agreed. Retention and compliance with all assessments was very high at all time-points. Only one patient was withdrawn from the study due to disease progression.

\section{Conclusions}

Findings from this study demonstrate that it is feasible to longitudinally assess cognitive status and impairment in people with newly diagnosed aggressive lymphoma during their initial treatment and recovery and larger studies should be undertaken within other cancer groups.

\section{Introduction}

Cancer related cognitive impairment $(\mathrm{CRCl})$ is a highly distressing and disabling side effect commonly reported by cancer patients. $[1,2]$ The incidence varies, but studies of patients with solid tumours suggest that up to $70 \%$ of patients receiving treatment self-report some degree of cognitive impairment. [1, 2] The cognitive domains most commonly affected are memory, concentration, information processing, speed and executive function [1]. For some patients cognitive impairment may be transient, but for a subgroup these symptoms can be long-lasting and have a major impact on quality of life and ability to function. [3]

Lymphoma is the 6th most common cancer in adults, and is the most common cancer in young people aged 15-29 years in Australia. [4] Aggressive lymphomas including Hodgkin lymphoma (HL) and non-Hodgkin lymphoma (NHL), such as diffuse large B-cell lymphoma (DLBCL) are potentially curable cancers. Current treatments, consisting of combination chemotherapy with or without radiotherapy, provide a 5-year progression-free survival (PFS) of $65-92 \%$ and potential cure in around $50 \%$ of patients. [5] This, coupled with a favourable prognosis, has resulted in a growing population of survivors of aggressive lymphoma, who are at risk of side-effects from the long-term toxicity associated with the treatments received. [6]

Persistent changes in cognitive function are frequently reported by lymphoma survivors. [7-9] The majority of CRCl studies have been performed in women with breast cancer, while several small-size studies incorporated patients with other solid tumours. [10-12] In contrast to aggressive lymphoma patients, these malignancies primarily affect older adults, hence they form the target population in the majority of $\mathrm{CRCl}$ studies. In young adults, with a longer life expectancy, impaired cognition may have a dramatic impact on their quality of life, affecting their working and learning capacities and multiple aspects of their social life. 
Despite such widespread implications, the feasibility of collecting longitudinal data on cognition in patients with aggressive lymphoma during treatment has not been explored.

The International Cognition and Cancer Task Force (ICCTF), formed with the goal of improving understanding of the impact cancer and its treatments have on cognition, recommends comprehensive, longitudinal neuropsychological assessment (NPA) as the gold standard for measuring cognitive function. [13] Emerging evidence suggests that blood-cell inflammatory markers may serve as a valuable prognostic indicator of cognitive impairment and susceptibility among cancer patients, $[14,15]$ and neuroimaging studies have demonstrated cerebral structural changes associated with chemotherapy. [16-19] Yet, few studies have assessed cognition in patients pre-and post-treatment, [16-18] and there are limited data of cognitive changes in patients with haematological malignancies. [20-22] Given the need for urgent comprehensive diagnostic work-up and rapid commencement of chemotherapy among patients with aggressive lymphoma, establishing the feasibility of longitudinal, and particularly pre-treatment NPA, is an important goal, if the recommendations of the ICCTF are to be realised. To date, a longitudinal exploration of the pattern of $\mathrm{CRCl}$ over the course of treatment and recovery has not been described in this population.

Given the limited data on the feasibility of comprehensively assessing cognition in patients with non-CNS aggressive lymphoma before, during, and after treatment it was important to explore this issue before embarking on a large-scale study to comprehensively describe the cognitive outcomes and trajectory of this patient cohort. We aimed to: (1) assess the feasibility of collecting longitudinal data on cognition using self-report measures and objective neuropsychological tests in people with newly diagnosed aggressive lymphoma undergoing standard therapy with curative intent, and (2) explore and describe patterns of $\mathrm{CRCl}$ in the population of interest as measured by self-report measures, neuropsychological tests, blood cell-based inflammatory markers and neuroimaging.

\section{Methods}

\section{Study design and setting}

This single-site, single-arm, longitudinal feasibility study was conducted in a specialised haematology department of a comprehensive cancer centre in a large acute hospital in metropolitan Melbourne, Australia. The published protocol provides a detailed description of study methods and procedures. [23] The study schema is presented in Figure 1.

\section{Participants}

Participants were 18 years or older with newly diagnosed aggressive lymphoma; scheduled to undergo standard combination chemotherapy with curative intent; able to read and comprehend English; and had a documented ECOG Performance Status 0 to 2. [24] Patients were not eligible if they had any of the following: lymphomatous CNS involvement, prior or planned cranial radiation therapy, a life expectancy less than 12 months, any medical condition that might compromise adherence or lead to prolonged hospitalisation, a documented history of past or current substance misuse, or poorly controlled psychiatric illness.

\section{Recruitment procedures}

Eligible patients were approached and invited to participate. [23] A copy of the participant information statement and consent form (PICF) was provided, then written informed consent obtained for self-report measures, neuropsychological tests, blood cellbased inflammatory markers and screening for eligibility for neuroimaging sub-study.

\section{Data collection procedures for all study data}

Consenting participants underwent comprehensive assessments at three pre-specified times: pre-chemotherapy (T1), midchemotherapy (T2), and 6-8 weeks post-chemotherapy (T3) (Figure 1). Neuropsychological tests included the Hopkins Verbal

Page $3 / 20$ 
Learning Test-Revised (HVLT-R), Controlled Oral Word Association (COWA), Stroop Colour and Word, Trail Making Test Part A\&B, and Digit Span (WAIS-R). Patient-reported outcome measures included the EORTC Quality of Life Questionnaire Cognitive functioning scale (QLQ-C30 CF), the Functional Assessment of Cancer Therapy-General (FACT-G) and -Cognitive Function (FACTCog), Cognitive Failures Questionnaire (CFQ), Functional Assessment of Chronic Illness Therapy-Fatigue (FACIT-F), and PROMIS Emotional Distress-Depression 8b and -Anxiety 7a short forms. A detailed description of each measure is provided in the protocol paper. [23]

Laboratory tests including full blood examination (FBE) were collected as part of standard care. These parameters were used to calculate blood cell-based inflammatory markers including the neutrophil to lymphocyte ratio (NLR), platelet to lymphocyte ratio (PLR), and systemic immune-inflammation index (SII). Neuroimaging ( ${ }^{18}$ F-FDG PET/CT brain acquisition study and MRI scan) formed part of an optional sub-study and the brain MRI sub-study occurred at two time points only. Patients who had already had their standard of care diagnostic PET scans before attending the lymphoma service were not eligible to participate in the sub-study. Interview data were collected to explore participant burden and a qualitative sub-study explored motivation to participate [25] (refer to Figure 1).

\section{Analysis}

Feasibility outcomes. Consent data were summarised using a proportion and $95 \%$ confidence intervals (Cl) estimated using the Wilson method. Recruitment data were summarised using a rate and $95 \% \mathrm{Cl}$ estimated using the Poisson distribution. Retention, compliance with assessments, and consent and assessment context data were summarised using counts and percentages.

Qualitative data. Audio-recorded interview data were transcribed verbatim and de-identified word documents produced. An interpretivist perspective (interpretive description) guided thematic analysis. [26] Interpretive description allows for investigation of a clinical phenomenon of interest, particularly in smaller studies, to capture themes and patterns associated with subjective perceptions, in order to inform clinical understanding. [26] The framework approach was used to apply initial codes to interview transcripts, [27] before following the six phases of analysis recommended by Braun and Clark (2006).[28] For a more complete description of analytic methods refer to the qualitative exploration previously published. [25]

Participant characteristics and study measures. Study measures were scored according to author guidelines. Descriptive statistics (counts and percentages; means, standard deviations and/or $95 \% \mathrm{Cl}$; medians and interquartile ranges; and ranges, as appropriate) were used to summarise participant characteristics, missing data, pre-chemotherapy study measure scores and score changes at follow-up assessments (T2 and T3) from pre-chemotherapy, as well as pre-chemotherapy blood cell-based inflammatory markers and changes in these markers at follow-up assessments (T2 and T3) from pre-chemotherapy. Confidence intervals for the latter were bootstrapped (10,000 replications) due to the small sample and observable skew. Kazis et al [29] effect size estimates were calculated to characterise the sizes of changes from pre-chemotherapy. Linear mixed models were used to assess the overall pattern of change in self-report measures and neuropsychological tests. All models included a fixed effect for time and random participant effect. All analysis was performed in R (version 3.6.1) [30], using 'epitools' [31], 'binom' [32], 'wBoot' [33] and 'Ime4' [34].

\section{Results}

\section{Study Profile}

Fifty-five patients with newly diagnosed aggressive lymphoma were screened for eligibility between 26 November 2019 and 01 September 2020. Twenty-two patients were ineligible. Reasons for ineligibility are summarised in Figure 2. Thirty-three patients met study eligibility criteria and were approached to participate. Thirty of 33 patients consented to participate. PET/CT brain scans were obtained for 11 of 30 participants; the remaining participants had had their diagnostic PET scans before attending the lymphoma service. 
Characteristics of the study sample pre-chemotherapy are summarised in Table 1 . The median age was 57 years (range 18 to 78 years) and 16/30 (53\%) participants were male. Most participants were diagnosed with DLBCL ( $\mathrm{n}=20,66 \%)$ or HL $(n=4 ; 14 \%)$. All participants diagnosed with aggressive lymphoma received combination chemotherapy as per standard of care. 
Participant characteristics $(n=30)$

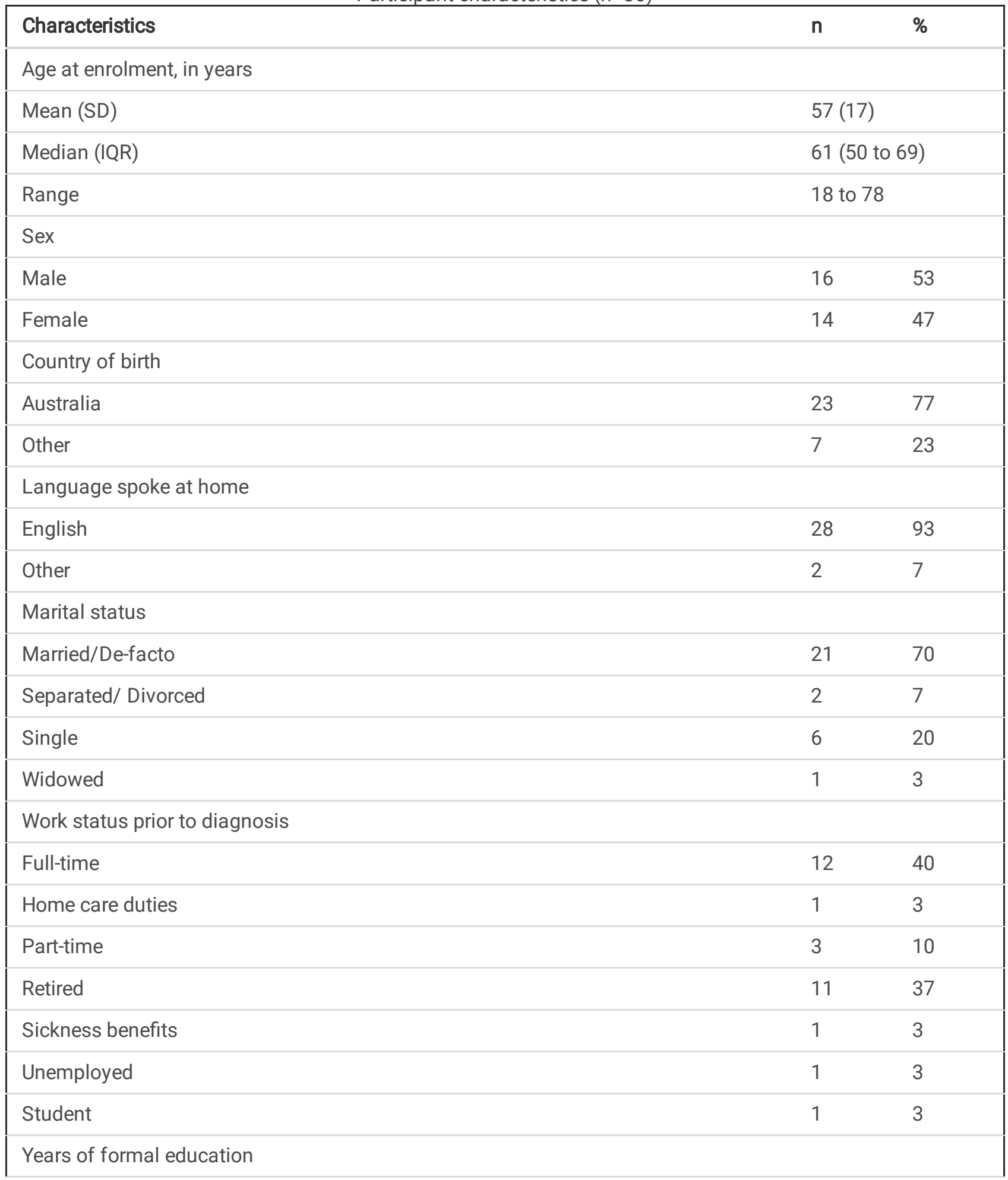

\section{Notes:}

ABVD: Adriamycin, bleomycin, vinblastine, dacarbazine.

R-CHOP: rituximab, cyclophosphamide, doxorubicin, vincristine, prednisolone.

ESC-BEACOPP: bleomycin, etoposide, doxorubicin, cyclophosphamide, vincristine, procarbazine, prednisolone

HD MTX: high-dose methotrexate

R-DHAP: rituximab, dexamethasone, cytarabine, cisplatin 


\begin{tabular}{|c|c|c|}
\hline Characteristics & $\mathbf{n}$ & $\%$ \\
\hline Mean (SD) & \multicolumn{2}{|c|}{$13(2)$} \\
\hline Median (IQR) & \multicolumn{2}{|c|}{$13(12$ to 14$)$} \\
\hline Range & \multicolumn{2}{|c|}{7 to 18} \\
\hline \multicolumn{3}{|l|}{ ECOG performance status } \\
\hline 0 & 21 & 70 \\
\hline 1 & 9 & 30 \\
\hline \multicolumn{3}{|l|}{ Colinet comorbidity score } \\
\hline Median (IQR) & \multicolumn{2}{|c|}{0 (0 to 1$)$} \\
\hline Range & \multicolumn{2}{|c|}{0 to 9} \\
\hline \multicolumn{3}{|c|}{ Active psychiatric / medication affecting cognition } \\
\hline No & 27 & 90 \\
\hline Yes & 3 & 10 \\
\hline \multicolumn{3}{|c|}{ Been treated for depression/ anxiety / psychiatric / neurological condition } \\
\hline No & 22 & 73 \\
\hline Yes & 8 & 27 \\
\hline Anxiety & 2 & \\
\hline Chronic schizophrenia & 1 & \\
\hline Depression & 4 & \\
\hline Panic attacks & 1 & \\
\hline \multicolumn{3}{|l|}{ Diagnosis } \\
\hline DLBCL & 20 & 67 \\
\hline Grade 3B Follicular Lymphoma & 1 & 3 \\
\hline $\mathrm{HL}$ & 4 & 13 \\
\hline Mantle cell Lymphoma & 1 & 3 \\
\hline T-cell Lymphoma & 3 & 10 \\
\hline Primary mediastinal B-cell Lymphoma & 1 & 3 \\
\hline \multicolumn{3}{|l|}{ Chemotherapy regimen } \\
\hline$A B V D \times 6$ & 3 & 10 \\
\hline \multicolumn{3}{|l|}{ Notes: } \\
\hline \multicolumn{3}{|c|}{ ABVD: Adriamycin, bleomycin, vinblastine, dacarbazine. } \\
\hline \multicolumn{3}{|c|}{ R-CHOP: rituximab, cyclophosphamide, doxorubicin, vincristine, prednisolone. } \\
\hline \multicolumn{3}{|c|}{ Esc-BEACOPP: bleomycin, etoposide, doxorubicin, cyclophosphamide, vincristine, procarbazine, prednisolone } \\
\hline \multicolumn{3}{|c|}{ HD MTX: high-dose methotrexate } \\
\hline R-DHAP: rituximab, dexamethasone, cy & & \\
\hline
\end{tabular}




\begin{tabular}{|c|c|c|}
\hline Characteristics & $\mathbf{n}$ & $\%$ \\
\hline $\mathrm{CHOP} \times 6$ & 2 & 7 \\
\hline Esc-BEACOPP x 4 & 1 & 3 \\
\hline Mini R-CHOP x 6 & 2 & 7 \\
\hline $\mathrm{R}-\mathrm{CHOP} \times 2$ & 1 & 3 \\
\hline $\mathrm{R}-\mathrm{CHOP} \times 3$ & 2 & 7 \\
\hline $\mathrm{R}-\mathrm{CHOP} \times 4$ & 3 & 10 \\
\hline $\mathrm{R}-\mathrm{CHOP} \times 6$ & 10 & 33 \\
\hline R-CHOP \& HD MTX x 2 & 1 & 3 \\
\hline R-CHOP \& R $\times 2$ & 4 & 13 \\
\hline R-CHOP/R-DHAP x 3 & 1 & 3 \\
\hline \multicolumn{3}{|c|}{ Length of chemotherapy treatment, in days } \\
\hline Mean (SD) & \multicolumn{2}{|c|}{$102(34)$} \\
\hline Median (IQR) & \multicolumn{2}{|c|}{105 (105 to 114$)$} \\
\hline Range & \multicolumn{2}{|c|}{21 to 116} \\
\hline \multicolumn{3}{|l|}{ Notes: } \\
\hline \multicolumn{3}{|c|}{ ABVD: Adriamycin, bleomycin, vinblastine, dacarbazine. } \\
\hline \multicolumn{3}{|c|}{ R-CHOP: rituximab, cyclophosphamide, doxorubicin, vincristine, prednisolone. } \\
\hline \multicolumn{3}{|c|}{ Esc-BEACOPP: bleomycin, etoposide, doxorubicin, cyclophosphamide, vincristine, procarbazine, prednisolone } \\
\hline \multicolumn{3}{|c|}{ HD MTX: high-dose methotrexate } \\
\hline \multicolumn{3}{|c|}{ R-DHAP: rituximab, dexamethasone, cytarabine, cisplatin } \\
\hline
\end{tabular}

\section{Feasibility Outcomes}

The recruitment rate was 3 patients/month (95\% Cl: 2.0, 4.3 patients/month) and the consent rate was $91 \%$ ( 30 of $33,95 \% \mathrm{Cl}$ : $76 \%, 97 \%$ ). Retention at follow-up was excellent (Figure 2); only one participant was withdrawn from the study between T2 and T3 as a consequence of disease progression.

\section{Feasibility of consent and timing of diagnosis}

The median time between patient informed of diagnosis and consent was 2 days (inter-quartile range: 0 to 7 days; range: 0 to 33 days). The median time between patient informed of diagnosis and pre-chemotherapy assessment was 7 days (inter-quartile range: 2 to 14 days; range: 0 to 49 days). The median time between patient informed of diagnosis and treatment commencement was 12 days (inter-quartile range: 7 to 17 days; range: 0 to 99 days).

\section{Consent and assessment context}

The context of consent and study assessments is summarised in Table 2. Most patients (20/30) were approached and invited to participate while attending the lymphoma outpatient clinic situated in the cancer centre; seven were consented as inpatients. Six participants completed their pre-chemotherapy NPA in the day oncology unit prior to commencing their chemotherapy infusion; six were in lymphoma outpatient clinic and six were inpatients on the haematology ward. Some participants (13/30) completed their mid-chemotherapy NPA on the day their PET scan was scheduled; ten completed this assessment in the day oncology unit 
prior to the commencement of their third cycle of chemotherapy. The majority of participants $(19 / 30)$ completed their 6-8 weeks post-chemotherapy NPA on the day their PET or MRI scan was scheduled.

Table 2

Consent and assessment context

\begin{tabular}{|c|c|c|}
\hline Context & $\mathbf{n}$ & $\%$ \\
\hline \multicolumn{3}{|l|}{ Consent, $n=30$} \\
\hline Day of Chemotherapy & 2 & 7 \\
\hline Day of chemotherapy education & 1 & 3 \\
\hline Lymphoma clinic & 20 & 67 \\
\hline Inpatient haematology & 5 & 17 \\
\hline Inpatient other & 2 & 7 \\
\hline \multicolumn{3}{|l|}{ Pre-chemotherapy assessment, $n=30$} \\
\hline Day of chemotherapy commence & 6 & 20 \\
\hline Day of chemotherapy education & 5 & 17 \\
\hline Inpatient & 6 & 20 \\
\hline Lymphoma clinic & 6 & 20 \\
\hline Post PET scan & 2 & 7 \\
\hline Post MRI scan & 3 & 10 \\
\hline Stand-alone appointment & 2 & 7 \\
\hline \multicolumn{3}{|l|}{ Mid-chemotherapy assessment, $n=30$} \\
\hline Day of chemotherapy & 10 & 33 \\
\hline Inpatient & 1 & 3 \\
\hline Pre-PET scan & 7 & 23 \\
\hline Lymphoma clinic & 5 & 17 \\
\hline Post PET scan & 6 & 20 \\
\hline Stand-alone appointment & 1 & 3 \\
\hline \multicolumn{3}{|c|}{ 6-8 weeks post-chemotherapy assessment, $n=29$} \\
\hline Lymphoma clinic & 4 & 14 \\
\hline Pre MRI scan & 1 & 3 \\
\hline Pre-PET scan & 9 & 31 \\
\hline Post PICC line removal & 3 & 10 \\
\hline Post PET scan & 9 & 31 \\
\hline Stand-alone appointment & 3 & 10 \\
\hline
\end{tabular}

\section{Compliance with assessments and accessibility of laboratory tests}

Compliance with study measures was high at all time points. For patient-reported outcome measures, compliance was $90-100 \%$ at T1, T2 and T3 (Table 3). For NPA, compliance was $100 \%$ at T1, T2 and T3 for all active participants (Table 4). Laboratory tests 
were available for all active participants at $\mathrm{T} 1, \mathrm{~T} 2$ and $\mathrm{T} 3$.

All 30 participants were screened for the neuroimaging sub-study. Nineteen participants were ineligible as the diagnostic standard of care whole-body PET/CT scan was completed prior to attending the lymphoma service. A total of eleven participants met the eligibility criteria and were invited to participate. All $(11 ; 100 \%)$ agreed to participate, however two participants declined MRI brain study due to claustrophobia and anxiety from previous MRI scans.

Compliance with the PET scans was $100 \%$ (11 of 11) at T1, T2, and T3 for eligible participants. Compliance with the MRI scans was 100\% (9 of 9) at T1 and 89\% (8 of 9) at T3 (Figure 2) for consenting participants. This is despite some participants describing the additional neuroimaging requirements as time-consuming, uncomfortable and anxiety provoking. One participant declined the final MRI due to distress related to disease progression. Disease progression was identified one week after other study measures were completed.

Several feasibility issues were identified in collection of the neuroimaging data. The MRI study included small sample size and was only performed at two timepoints. For the PET scans there was large time delays between scan acquisition and FDG tracer injection in five patients, variability in the scanner used, and variability in days between $\mathrm{T} 1$ and follow-up imaging. Therefore, we have addressed and reported feasibility objectives only.

\section{Acceptability of study measures}

The first five participants were invited to take part in a participant burden interview one week after the pre-chemotherapy assessment to assess the acceptability of the study measures. Three participants found specific neuropsychological tests difficult and two found the assessments tiring. Nevertheless, none of the participants recommended changes to the assessment schedule. [35]

\section{Patient-reported outcome measures and neuropsychological assessments}

Descriptive statistics for pre-chemotherapy patient-reported outcome measures and neuropsychological test scores, as well as changes from pre-chemotherapy at follow-up assessments (T2 and T3) are provided in Tables 3 and 4, respectively. Linear mixed model results are provided in online supplemental Appendices A and B for completeness.

Small- to medium-sized changes were observed on a number of patient-reported outcome measure scale/total scores (Table 3); for the most part, changes reflected a deterioration in relevant domains at follow-up, apart from changes in anxiety and depression, which indicated improvement at follow-up assessments. 
Table 3

Descriptive statistics for patient-reported outcome measures, pre-chemotherapy scores and mean changes at follow-up assessments

\begin{tabular}{|c|c|c|c|c|c|c|c|c|c|c|c|c|c|}
\hline \multirow[t]{2}{*}{ Measure } & \multicolumn{3}{|c|}{ Pre-chemotherapy } & \multicolumn{5}{|c|}{ Mid-chemotherapy } & \multicolumn{5}{|c|}{ 6-8 weeks post-chemotherapy } \\
\hline & $\mathrm{n}$ & M & SD & $n$ & M & SD & $\begin{array}{l}M \text { chg } \\
(95 \% \\
\text { Cl) }\end{array}$ & ES & $n$ & M & SD & $\begin{array}{l}\text { M } \\
\text { chg } \\
(95 \% \\
\mathrm{Cl})\end{array}$ & ES \\
\hline \multicolumn{14}{|l|}{$\begin{array}{l}\text { EORTC QLQ-C30 } \\
\text { Cognitive functioning }\end{array}$} \\
\hline Total score & 30 & 0.9 & 1.2 & 30 & 1.3 & 1.7 & $\begin{array}{l}0.4 \\
(-0.2 \\
1.0)\end{array}$ & 0.34 & 29 & 1.3 & 1.1 & $\begin{array}{l}0.4( \\
-0.1, \\
0.9)\end{array}$ & 0.35 \\
\hline \multicolumn{14}{|l|}{ FACT-Cog } \\
\hline $\begin{array}{l}\text { Perceived cognitive } \\
\text { impairment }\end{array}$ & 30 & 60.8 & 11.9 & 30 & 56.2 & 16.2 & $\begin{array}{l}-4.6 \\
(-8.3 \\
-0.9)\end{array}$ & 0.39 & 29 & 57.1 & 15.3 & $\begin{array}{l}-4.2( \\
-8.1 \\
-0.2)\end{array}$ & 0.35 \\
\hline $\begin{array}{l}\text { Impact of perceived } \\
\text { impairment on QOL }\end{array}$ & 30 & 11.3 & 4.7 & 29 & 11.9 & 4.4 & $\begin{array}{l}0.8 \\
(-0.7 \\
2.2)\end{array}$ & 0.16 & 29 & 11.6 & 4.4 & $\begin{array}{l}0.5( \\
-1.4, \\
2.4)\end{array}$ & 0.10 \\
\hline $\begin{array}{l}\text { Perceived cognitive } \\
\text { abilities }\end{array}$ & 30 & 21.7 & 6.2 & 29 & 18.7 & 8.4 & $\begin{array}{l}-3.0 \\
(-6.0 \\
0.0)\end{array}$ & 0.48 & 29 & 19.4 & 7.1 & $\begin{array}{l}-2.5( \\
-4.4 \\
-0.5)\end{array}$ & 0.39 \\
\hline \multicolumn{14}{|l|}{$\begin{array}{l}\text { Cognitive Failures } \\
\text { Questionnaire }\end{array}$} \\
\hline Forgetfulness & 30 & 9.6 & 4.9 & & & & & & 29 & 10.3 & 5.2 & $\begin{array}{l}0.7( \\
-0.8, \\
2.2)\end{array}$ & 0.14 \\
\hline Distractibility & 30 & 9.2 & 5.1 & & & & & & 29 & 9.5 & 5.5 & $\begin{array}{l}0.5( \\
-1.1 \\
2.1)\end{array}$ & 0.09 \\
\hline False Triggering & 30 & 5.8 & 4.1 & & & & & & 29 & 7.1 & 4.9 & $\begin{array}{l}1.3( \\
-0.3 \\
3.0)\end{array}$ & 0.33 \\
\hline \multicolumn{14}{|l|}{ FACT-G } \\
\hline Physical wellbeing & 29 & 21.2 & 6.3 & 30 & 19.4 & 7.2 & $\begin{array}{l}-1.8( \\
-4.8 \\
1.1)\end{array}$ & 0.29 & 29 & 20.6 & 5.5 & $\begin{array}{l}-1.3( \\
-3.8 \\
1.2)\end{array}$ & 0.20 \\
\hline Social wellbeing & 29 & 23.2 & 4.9 & 29 & 23.4 & 5.0 & $\begin{array}{l}-0.2( \\
-1.1 \\
0.8)\end{array}$ & 0.03 & 28 & 23.4 & 5.2 & $\begin{array}{l}0.1( \\
-1.1, \\
1.3)^{\prime}\end{array}$ & 0.02 \\
\hline Emotional wellbeing & 28 & 16.4 & 5.1 & 30 & 18.8 & 4.1 & $\begin{array}{l}2.4( \\
0.5,4.2)\end{array}$ & 0.47 & 29 & 19.3 & 3.8 & $\begin{array}{l}2.9( \\
1.3 \\
4.4)\end{array}$ & 0.56 \\
\hline \multicolumn{14}{|l|}{ Notes: } \\
\hline \multicolumn{14}{|c|}{$\begin{array}{l}\text { M chg: mean change at follow-up assessments from baseline (interpretation of } \mathrm{M} \text { chg for measures described below); Cl: } \\
\text { confidence interval; ES: Kazis effect size (interpretation of mean changes: } 0.2 \text {, small; } 0.5 \text {, medium; and } 0.8, \text { large), medium- } \\
\text { sized changes are italicised and large-sized changes are in bold for emphasis. }\end{array}$} \\
\hline \multicolumn{14}{|c|}{$\begin{array}{l}\text { Interpretation of M chg: for the EORTC Cognitive functioning scale Total score, FACT-Cog scales, Cognitive Failures } \\
\text { Questionnaire scales, and PROMIS Emotional Distress measures, a positive M chg reflects deterioration, and a negative M } \\
\text { chg reflects improvement; for the FACT-G domain and total scores, and FACIT-F Total score, a positive M chg reflects } \\
\text { improvement, and a negative M chg reflects deterioration. }\end{array}$} \\
\hline
\end{tabular}




\begin{tabular}{|c|c|c|c|c|c|c|c|c|c|c|c|c|c|}
\hline \multirow{2}{*}{$\begin{array}{l}\text { Measure } \\
\text { Functional wellbeing }\end{array}$} & \multicolumn{3}{|c|}{ Pre-chemotherapy } & \multicolumn{5}{|c|}{ Mid-chemotherapy } & \multicolumn{5}{|c|}{ 6-8 weeks post-chemotherapy } \\
\hline & 28 & 19.5 & 6.2 & 30 & 18.1 & 6.0 & $\begin{array}{l}-1.6( \\
-3.6 \\
0.4)\end{array}$ & 0.25 & 29 & 18.1 & 5.9 & $\begin{array}{l}-1.4 \\
(-3.2 \\
0.4)\end{array}$ & 0.23 \\
\hline Total score & 28 & 80.2 & 15.6 & 29 & 79.6 & 16.0 & $\begin{array}{l}-1.1 \\
(-6.5 \\
4.3)\end{array}$ & 0.07 & 28 & 81.4 & 15.2 & $\begin{array}{l}0.3 \\
(-4.6 \\
5.1)\end{array}$ & 0.02 \\
\hline \multicolumn{14}{|l|}{ FACIT-F } \\
\hline Total score & 27 & 37.3 & 13.3 & 30 & 33.8 & 12.4 & $\begin{array}{l}-3.7 \\
(-7.8 \\
0.4)\end{array}$ & 0.10 & 29 & 32.6 & 11.5 & $\begin{array}{l}-5.6( \\
-9.7 \\
-1.6)\end{array}$ & 0.42 \\
\hline \multicolumn{14}{|l|}{$\begin{array}{l}\text { PROMIS Emotional } \\
\text { Distress }\end{array}$} \\
\hline Depression 8b & 28 & 51.5 & 8.3 & 27 & 50.3 & 10.4 & $\begin{array}{l}-1.7( \\
-4.7 \\
1.3)\end{array}$ & 0.21 & 27 & 49.0 & 9.1 & $\begin{array}{l}-2.3( \\
-5.2, \\
0.6)\end{array}$ & 0.28 \\
\hline Anxiety $7 a$ & 28 & 55.8 & 9.9 & 29 & 49.1 & 10.3 & $\begin{array}{l}-6.6 \\
(-10.1 \\
-3.2)\end{array}$ & 0.67 & 27 & 48.2 & 9.4 & $\begin{array}{l}-7.4( \\
-10.7 \\
-4.0)\end{array}$ & 0.74 \\
\hline \multicolumn{14}{|l|}{ Notes: } \\
\hline \multicolumn{14}{|c|}{$\begin{array}{l}\text { M chg: mean change at follow-up assessments from baseline (interpretation of } \mathrm{M} \text { chg for measures described below); } \mathrm{Cl} \text { : } \\
\text { confidence interval; ES: Kazis effect size (interpretation of mean changes: } 0.2 \text {, small; } 0.5 \text {, medium; and } 0.8 \text {, large), medium- } \\
\text { sized changes are italicised and large-sized changes are in bold for emphasis. }\end{array}$} \\
\hline \multicolumn{14}{|c|}{$\begin{array}{l}\text { Interpretation of M chg: for the EORTC Cognitive functioning scale Total score, FACT-Cog scales, Cognitive Failures } \\
\text { Questionnaire scales, and PROMIS Emotional Distress measures, a positive M chg reflects deterioration, and a negative M } \\
\text { chg reflects improvement; for the FACT-G domain and total scores, and FACIT-F Total score, a positive M chg reflects } \\
\text { improvement, and a negative M chg reflects deterioration. }\end{array}$} \\
\hline
\end{tabular}

Small- to medium-sized changes were observed on some neuropsychological test scale/total scores (Table 4); for the most part, changes suggested improvement at follow-up assessments. 
Table 4

Descriptive statistics for neuropsychological tests, pre-chemotherapy scores and mean changes at follow-up assessments

\begin{tabular}{|c|c|c|c|c|c|c|c|c|c|c|c|c|c|}
\hline \multirow[t]{2}{*}{ Measure } & \multicolumn{3}{|c|}{ Pre-chemotherapy } & \multicolumn{5}{|c|}{ Mid-chemotherapy } & \multicolumn{5}{|c|}{ 6-8 weeks post-chemotherapy } \\
\hline & $\mathrm{n}$ & M & SD & $\mathrm{n}$ & M & SD & $\begin{array}{l}\text { M } \\
\text { diff } \\
(95 \% \\
\mathrm{Cl})\end{array}$ & ES & $\mathrm{n}$ & M & SD & $\begin{array}{l}\text { M } \\
\text { diff } \\
(95 \% \\
\mathrm{Cl})\end{array}$ & ES \\
\hline \multicolumn{14}{|l|}{$\begin{array}{l}\text { Stroop Colour and Word } \\
\text { Test }\end{array}$} \\
\hline Colour & 30 & 43.8 & 9.1 & 30 & 43.0 & 10.7 & $\begin{array}{l}-0.8( \\
-2.9 \\
1.2)\end{array}$ & 0.09 & 29 & 41.7 & 9.0 & $\begin{array}{l}-2.2( \\
-4.3 \\
-0.1)\end{array}$ & 0.24 \\
\hline Word & 30 & 43.0 & 10.9 & 30 & 42.9 & 10.1 & $\begin{array}{l}-0.2( \\
-3.1, \\
2.8)\end{array}$ & 0.02 & 29 & 42.6 & 9.8 & $\begin{array}{l}-0.7( \\
-4.2 \\
2.8)\end{array}$ & 0.06 \\
\hline Word/Colour & 30 & 48.2 & 10.3 & 30 & 48.6 & 9.3 & $\begin{array}{l}0.4( \\
-2.3 \\
3.1)\end{array}$ & 0.04 & 29 & 48.2 & 8.6 & $\begin{array}{l}0.1 \\
(-2.9 \\
3.1)\end{array}$ & 0.01 \\
\hline Inference Colour/Word & 30 & 46.8 & 8.9 & 30 & 48.2 & 8.8 & $\begin{array}{l}1.4 \\
(-1.3 \\
4.1)\end{array}$ & 0.16 & 29 & 48.1 & 8.2 & $\begin{array}{l}1.7( \\
-1.0 \\
4.4)\end{array}$ & 0.19 \\
\hline \multicolumn{14}{|l|}{ Trail Making Test } \\
\hline A score & 30 & 45.8 & 9.6 & 30 & 47.6 & 8.8 & $\begin{array}{l}1.8( \\
-0.7 \\
4.3)\end{array}$ & 0.19 & 29 & 48.6 & 9.6 & $\begin{array}{l}3.1( \\
-0.3 \\
6.5)\end{array}$ & 0.32 \\
\hline B score & 30 & 46.6 & 13.8 & 30 & 49.0 & 14.1 & $\begin{array}{l}2.4( \\
-0.4 \\
5.2)\end{array}$ & 0.17 & 29 & 47.3 & 14.1 & $\begin{array}{l}0.7( \\
-4.2 \\
5.6)\end{array}$ & 0.05 \\
\hline \multicolumn{14}{|l|}{$\begin{array}{l}\text { Hopkins Verbal Learning } \\
\text { Test }\end{array}$} \\
\hline Total Recall & 30 & 40.8 & 10.0 & 30 & 44.9 & 9.6 & $\begin{array}{l}4.1( \\
0.0 \\
8.2)\end{array}$ & 0.40 & 29 & 45.9 & 11.8 & $\begin{array}{l}5.2( \\
1.1, \\
9.3)\end{array}$ & 0.52 \\
\hline Delayed Recall & 30 & 38.1 & 12.4 & 30 & 41.7 & 12.7 & $\begin{array}{l}3.5( \\
-1.8 \\
8.9)\end{array}$ & 0.28 & 29 & 41.6 & 12.5 & $\begin{array}{l}3.6( \\
-1.5 \\
8.7)\end{array}$ & 0.29 \\
\hline Retention & 30 & 41.8 & 13.6 & 30 & 43.2 & 14.0 & $\begin{array}{l}1.4( \\
-5.8 \\
8.6)\end{array}$ & 0.10 & 29 & 41.9 & 12.2 & $\begin{array}{l}-0.1( \\
-6.1 \\
5.9)\end{array}$ & 0.01 \\
\hline Recognition/Discrimination & 30 & 46.7 & 11.6 & 30 & 39.3 & 14.9 & $\begin{array}{l}-7.4( \\
-12.6 \\
-2.2)\end{array}$ & 0.64 & 29 & 47.9 & 10.3 & $\begin{array}{l}1.5( \\
-2.5 \\
5.4)\end{array}$ & 0.13 \\
\hline
\end{tabular}

Notes:

$\mathrm{M}$ chg: mean change at follow-up assessments from baseline (interpretation of $\mathrm{M}$ chg for measures described below); $\mathrm{Cl}$ : confidence interval; ES: Kazis effect size (interpretation of mean changes: 0.2 , small; 0.5 , medium; and 0.8, large), mediumsized changes are italicised and large-sized changes are in bold for emphasis.

Interpretation of $\mathrm{M}$ chg: for all measures, a positive $\mathrm{M}$ chg reflects an improvement in cognitive performance, and a negative $\mathrm{M}$ chg reflects a deterioration in cognitive performance. 


\begin{tabular}{|c|c|c|c|c|c|c|c|c|c|c|c|c|c|}
\hline \multirow{2}{*}{$\begin{array}{l}\text { Measure } \\
\text { Total Letter Fluency }\end{array}$} & \multicolumn{3}{|c|}{ Pre-chemotherapy } & \multicolumn{5}{|c|}{ Mid-chemotherapy } & \multicolumn{5}{|c|}{ 6-8 weeks post-chemotherapy } \\
\hline & 30 & 42.3 & 11.9 & 30 & 48.2 & 11.4 & $\begin{array}{l}5.9( \\
2.3 \\
9.5)\end{array}$ & 0.50 & 29 & 46.0 & 12.2 & $\begin{array}{l}3.4( \\
-0.2 \\
7.1)\end{array}$ & 0.29 \\
\hline Category Fluency & 30 & 46.5 & 10.5 & 30 & 45.8 & 12.4 & $\begin{array}{l}-0.7( \\
-3.6 \\
2.3)\end{array}$ & 0.06 & 29 & 46.5 & 10.6 & $\begin{array}{l}0.0( \\
-2.9 \\
2.8)\end{array}$ & 0.00 \\
\hline Total Written Fluency & 30 & 44.5 & 11.7 & 30 & 46.7 & 14.4 & $\begin{array}{l}2.2( \\
-0.7 \\
5.0)\end{array}$ & 0.19 & 29 & 48.7 & 15.2 & $\begin{array}{l}4.1( \\
1.4 \\
6.8)\end{array}$ & 0.35 \\
\hline \multicolumn{14}{|c|}{$\begin{array}{l}\text { Digit Span Wechsler Adult } \\
\text { Intelligence Scale }\end{array}$} \\
\hline Digit Span Total & 30 & 48.2 & 8.1 & 30 & 50.1 & 9.9 & $\begin{array}{l}1.8( \\
-0.6 \\
4.3)\end{array}$ & 0.23 & 29 & 49.5 & 9.1 & $\begin{array}{l}1.1( \\
-1.1 \\
3.4)\end{array}$ & 0.14 \\
\hline \multicolumn{14}{|l|}{ Notes: } \\
\hline \multicolumn{14}{|c|}{$\begin{array}{l}\text { M chg: mean change at follow-up assessments from baseline (interpretation of } \mathrm{M} \text { chg for measures described below); Cl: } \\
\text { confidence interval; ES: Kazis effect size (interpretation of mean changes: } 0.2 \text {, small; } 0.5 \text {, medium; and 0.8, large), medium- } \\
\text { sized changes are italicised and large-sized changes are in bold for emphasis. }\end{array}$} \\
\hline
\end{tabular}

\section{Blood-cell based inflammatory markers}

Descriptive statistics for pre-chemotherapy blood cell-based inflammatory markers and changes in these markers at follow-up assessments are provided in online supplemental Appendix C for completeness. Small- to medium-sized changes were observed on the NLR and PLR, with higher blood-cell inflammatory markers reflecting lower cognitive performance. [15]

\section{Motivation for participation}

Four themes were generated from the qualitative interview data describing participants motivation for sustained participation in this study, at the time of heightened stress related to a new diagnosis of aggressive lymphoma: 1) ease of participation; 2) personal values that impact attitude to participation; 3) desire to engage in self-help; and 4) appreciation of additional support. Data and insights from the qualitative sub-study have been published earlier. [25]

\section{Discussion}

Our study recommends that it is feasible to complete a comprehensive assessment of cognitive outcomes in 30 patients with newly diagnosed aggressive lymphoma over the course of treatment and recovery.

Despite the distressing, challenging and stressful nature of the lymphoma diagnosis, recruitment to our study exceeded our expectations, with 30 patients with newly diagnosed aggressive lymphoma recruited over a 10-month period, and only three people declining participation. Participants may have been motivated to take part to help others. Results describing motivation and reasons for sustained participation have been published previously. [25]

Patients willing to participate in the study were often consented within days of diagnosis. Thirteen (43\%) were consented on day of being informed of diagnosis. Consent was obtained in a variety of clinical settings, with seven (23\%) participants being inpatients, five in the dedicated haematology ward, and two in general surgical wards. The median time between being informed of diagnosis and pre-chemotherapy NPA assessment was 7 days (range 0 to 49), with 4 (13\%) participants informed of diagnosis, consented to the study and assessed on the same day. The environment for collection of neuropsychological data was flexible and included a variety of clinical settings (e.g., day oncology unit, outpatient clinic, or inpatient unit). Management of 
potential disruption by clinical staff in the inpatient setting was minimised by placing a 'do not disturb' sign on door. The median time between being informed of diagnosis and date of treatment commencement was 12 days (range 0 to 99); with one (3\%) participant being informed of diagnoses, consented, assessed and commencing treatment on the same day.

Retention and compliance with all measures was excellent, despite literature reporting challenges with recruitment of patients who have recently been diagnosed with cancer. [35] Other studies have reported that retention of participants to longitudinal studies can be challenging, [36-38] and attrition is often attributed to poor study design. [38, 39] A recent study by Janelsins et al. (2021) assessed longitudinal changes in cognition in patients with lymphoma before and after chemotherapy; however, they did not include a mid-treatment assessment. They experienced significant attrition throughout the study; retention immediately post-chemotherapy was $86 \%$, and $72 \% 6$ months post-chemotherapy, with losses to follow-up being the main reason for attrition. [9] This may have been multifactorial and exacerbated by there being no mid-treatment assessment, potentially causing lack of interest or motivation for participants to stay engaged.

Success with recruitment and compliance with assessments in the current study was likely due to flexibility in testing time and location, reflecting the availability of the study nurse across all clinical settings. In particular, this flexibility enabled study assessments to take place at times convenient to the participants, usually when they were already at the hospital, thereby reducing any additional travel demands. Importantly, participants were approached at the hospital by the study nurse, possibly contributing to the high recruitment rate. The study nurse may have been seen as a trustworthy credible member of the team. [36] While not involved in clinical care at the time of diagnosis, the study nurse responsible for recruiting patients and conducting assessments was an experienced haematology nurse and a clinician nurse-researcher. [23] As such she was in a privileged position, being a long-standing staff member in the clinical setting, which may have strengthened the clinical team's motivation to identify and refer patients to the study. [37] Additionally, potential participants likely appreciated the study nurse's clinical expertise, and this may have improved trust among patients, encouraging participation. [38-40] These observations highlight the potential benefit of clinician involvement in data collection, [26] strengthening capacity for clinical research, notwithstanding the related ethical concerns of the study nurse-participants relationship grounded in trust but open to participant coercion.

Interview studies were conducted to examine the acceptability of the study protocol. Initially, a participant burden interview was undertaken with the first five participants, where no changes to the assessment schedule were recommended by any participant. [35] Feasibility of the study was later confirmed via a separate qualitative sub-study that explored the motivations for initial and ongoing participation at a time of heightened stress related to a new diagnosis of aggressive lymphoma and the rapid commencement of treatment. Ease of participation; personal values that impact attitude to participation; desire to engage in selfhelp; and appreciation of additional support motivated ongoing participation. [25]

Despite all eligible participants agreeing to participate in the neuroimaging sub-study, only 11 of 30 (37\%) were deemed eligible as diagnostic PET/CT scans had been acquired prior to attending the lymphoma service for the other patients. Our results speak to the difficulty of capturing baseline neuroimaging assessments in all patients, however, in those who were eligible for the substudy it was feasible. We found challenges with collecting longitudinal research quality PET/CT brain acquisition studies, which require constraints on scan time acquisition and camera availability, to assess any biomarker change over time, was difficult to implement in a clinical setting. Notwithstanding excellent compliance among those who were eligible, some participants found the brain scans to be challenging, arduous and time-consuming, and thus these data should only be collected in future studies if critical, given reports of participant burden.

This study has limitations that impact the generalisability of results. The sample size was small, and participants were all recruited from a single institution. Furthermore, despite the high proportion of patients consenting to participate and the involvement of a haematology nurse specialist may limit replicability in other centres. There were several challenges identified in the neuroimaging sub-study which were detailed in the results section. We recommend strong collaboration with the PET imaging centre, and longer imaging slots for each participant. However, this may increase costs associated with imaging and potentially place more demands on participants, who are already under considerable stress. Future studies should aim to recruit a larger patient cohort from across multiple institutions and should include patients from different cancer groups. 


\section{Conclusion}

Findings from this study demonstrate that it is feasible to assess cognitive status in people with newly diagnosed aggressive lymphoma during their initial treatment. Our recruitment to the study despite being a time of heightened stress was excellent. Compliance and retention with all study measures including patient-reported outcome measures, NPA, laboratory tests and neuroimaging in eligible participants, was very high at all timepoints. Our results suggest that longitudinal assessment of cognitive function in patients during treatment and recovery is acceptable to the patients and therefore feasible. However, some of the data capture strategies were onerous and research quality PET scans were difficult to acquire in clinical settings and requires careful consideration before including in future studies. We recommend future large-scale studies should be undertaken to comprehensively describe cognitive outcomes and trajectory including larger patient groups within other cancer groups.

\section{Declarations}

Author contribution: PG contributed to the literature reviews and study design, was involved in all aspects of data analysis and the overall preparation and writing of the manuscript. She is undertaking this research as part of her PhD. MK is PG's principal $\mathrm{PhD}$ supervisor. KG, HD and MK have led the development and contributed to all aspects of the study, including design, data analysis, manuscript preparation and revision. KG, $\mathrm{HD}, \mathrm{MK}$ and $\mathrm{CW}$ contributed to the original concept for this study and have participated in all aspects of the design, research questions, methodology, data analysis, manuscript preparation and revision. $\mathrm{EH}, \mathrm{VD}, \mathrm{CR}, \mathrm{YP}, \mathrm{TC}$ and AW have contributed to the study's methodology, data analysis plan, manuscript preparation and revision. $J V$ and MD have contributed to the study's methodology, manuscript preparation and revision. All authors have been involved in drafting and critical evaluation of this manuscript. All authors have read and approved the final version.

Funding: This study is supported by a non-restricted educational grant from Celgene Pty Ltd to support the costs associated with the neuroimaging. A PhD scholarship to the first author is provided by the Olivia Newton-John Cancer Wellness and Research Centre Supportive Care Research PhD scholarship through the Victorian Cancer Agency.

Data availability: De-identified data supporting the findings of this study are available from the corresponding author upon request.

Code availability: NA

Ethics approval: Ethical approval has been granted by Austin Health Human Rights Ethics Committee (HREC) in Victoria Australia. This study was conducted in compliance with the principles of the Declaration of Helsinki (2013) and the principles of Good Clinical Practice and the Australian National Statement on Ethical Conduct in Human Research.

Trial registration number: Australian New Zealand Clinical Trials Registry ACTRN12619001649101.

Conflict of interest: The authors declare no competing interests.

Consent to participate: Informed consent was obtained from all individual participants included in the study.

Consent for publication: Participants signed informed consent regarding publishing their data.

\section{References}

1. T. A. Ahles et al., "Neuropsychologic impact of standard-dose systemic chemotherapy in long-term survivors of breast cancer and lymphoma," J Clin Oncol, vol. 20, no. 2, pp. 485-93, Jan 15 2002, doi: 10.1200/JC0.2002.20.2.485.

2. V. J. Bray, H. M. Dhillon, and J. L. Vardy, "Systematic review of self-reported cognitive function in cancer patients following chemotherapy treatment," J Cancer Surviv, vol. 12, no. 4, pp. 537-559, Aug 2018, doi: 10.1007/s11764-018-0692-x.

3. N. Tchen et al., "Cognitive function, fatigue, and menopausal symptoms in women receiving adjuvant chemotherapy for breast cancer," J Clin Oncol, vol. 21, no. 22, pp. 4175-83, Nov 15 2003, doi: 10.1200/JC0.2003.01.119.

Page 16/20 
4. Australian Institute of Health and Welfare. "Cancer data in Australia." https://www.aihw.gov.au/reports/cancer/cancer-datain-australia/contents/summary (accessed.

5. P. Johnson et al., "Adapted Treatment Guided by Interim PET-CT Scan in Advanced Hodgkin's Lymphoma," N Engl J Med, vol. 374, no. 25, pp. 2419-29, Jun 23 2016, doi: 10.1056/NEJMoa1510093.

6. D. J. Straus, "Long-term survivorship at a price: late-term, therapy-associated toxicities in the adult hodgkin lymphoma patient," Ther Adv Hematol, vol. 2, no. 2, pp. 111-9, Apr 2011, doi: 10.1177/2040620711402414.

7. K. Behringer et al., "Cancer-Related Fatigue in Patients With and Survivors of Hodgkin Lymphoma: The Impact on Treatment Outcome and Social Reintegration," J Clin Oncol, vol. 34, no. 36, pp. 4329-4337, Dec 20 2016, doi: 10.1200/JCO.2016.67.7450.

8. A. M. Williams, C. S. Zent, and M. C. Janelsins, "What is known and unknown about chemotherapy-related cognitive impairment in patients with haematological malignancies and areas of needed research," $\mathrm{Br} J$ Haematol, vol. 174, no. 6, pp. 835-46, Sep 2016, doi: 10.1111/bjh.14211.

9. M. C. Janelsins et al., "Longitudinal Changes in Cognitive Function in a Nationwide Cohort Study of Patients with Lymphoma Treated with Chemotherapy," J Nat/ Cancer Inst, Jul 13 2021, doi: 10.1093/jnci/djab133.

10. C. Anderson-Hanley, M. L. Sherman, R. Riggs, V. B. Agocha, and B. E. Compas, "Neuropsychological effects of treatments for adults with cancer: a meta-analysis and review of the literature," J Int Neuropsychol Soc, vol. 9, no. 7, pp. 967-82, Nov 2003, doi: 10.1017/S1355617703970019.

11. J. S. Wefel, S. R. Kesler, K. R. Noll, and S. B. Schagen, "Clinical characteristics, pathophysiology, and management of noncentral nervous system cancer-related cognitive impairment in adults," CA Cancer J Clin, vol. 65, no. 2, pp. 123-38, Mar 2015, doi: 10.3322/caac.21258.

12. J. L. Vardy et al., "Cognitive Function in Patients With Colorectal Cancer Who Do and Do Not Receive Chemotherapy: A Prospective, Longitudinal, Controlled Study," J Clin Oncol, vol. 33, no. 34, pp. 4085-92, Dec 1 2015, doi: 10.1200/JCO.2015.63.0905.

13. J. S. Wefel, J. Vardy, T. Ahles, and S. B. Schagen, "International Cognition and Cancer Task Force recommendations to harmonise studies of cognitive function in patients with cancer," Lancet Oncol, vol. 12, no. 7, pp. 703-8, Jul 2011, doi: 10.1016/S1470-2045(10)70294-1.

14. A. Walker, "Aspirin blocks cancer-associated cognitive impairment. Poster presentation," presented at the UCLA, Los Angeles, California, 2017.

15. K. D. van der Willik, V. Koppelmans, M. Hauptmann, A. Compter, M. A. Ikram, and S. B. Schagen, "Inflammation markers and cognitive performance in breast cancer survivors 20 years after completion of chemotherapy: a cohort study," Breast Cancer Res, vol. 20, no. 1, p. 135, Nov 15 2018, doi: 10.1186/s13058-018-1062-3.

16. B. C. McDonald, S. K. Conroy, T. A. Ahles, J. D. West, and A. J. Saykin, "Alterations in brain activation during working memory processing associated with breast cancer and treatment: a prospective functional magnetic resonance imaging study," $J$ Clin Oncol, vol. 30, no. 20, pp. 2500-8, Jul 10 2012, doi: 10.1200/JC0.2011.38.5674.

17. S. Deprez et al., "Longitudinal assessment of chemotherapy-induced structural changes in cerebral white matter and its correlation with impaired cognitive functioning," J Clin Oncol, vol. 30, no. 3, pp. 274-81, Jan 20 2012, doi: 10.1200/JC0.2011.36.8571.

18. S. K. Conroy et al., "Alterations in brain structure and function in breast cancer survivors: effect of post-chemotherapy interval and relation to oxidative DNA damage," Breast Cancer Res Treat, vol. 137, no. 2, pp. 493-502, Jan 2013, doi: 10.1007/s10549-012-2385-x.

19. S. Deprez et al., "Chemotherapy-induced structural changes in cerebral white matter and its correlation with impaired cognitive functioning in breast cancer patients," Hum Brain Mapp, vol. 32, no. 3, pp. 480-93, Mar 2011, doi: 10.1002/hbm.21033.

20. B. Baudino et al., "The chemotherapy long-term effect on cognitive functions and brain metabolism in lymphoma patients," $Q$ J Nucl Med Mol Imaging, vol. 56, no. 6, pp. 559-68, Dec 2012. [Online]. Available: https://www.ncbi.nlm.nih.gov/pubmed/23172518.

Page $17 / 20$ 
21. D. D. Correa et al., "A prospective evaluation of changes in brain structure and cognitive functions in adult stem cell transplant recipients," Brain Imaging Behav, vol. 7, no. 4, pp. 478-90, Dec 2013, doi: 10.1007/s11682-013-9221-8.

22. D. D. Correa et al., "Prospective assessment of white matter integrity in adult stem cell transplant recipients," Brain Imaging Behav, vol. 10, no. 2, pp. 486-96, Jun 2016, doi: 10.1007/s11682-015-9423-3.

23. P. Gates et al., "Longitudinal exploration of cancer-related cognitive impairment in patients with newly diagnosed aggressive lymphoma: protocol for a feasibility study," BMJ Open, vol. 10, no. 9, p. e038312, Sep 29 2020, doi: 10.1136/bmjopen-2020038312.

24. M. M. Oken et al., "Toxicity and response criteria of the Eastern Cooperative Oncology Group," Am J Clin Oncol, vol. 5, no. 6, pp. 649-55, Dec 1982. [Online]. Available: https://www.ncbi.nlm.nih.gov/pubmed/7165009.

25. P. Gates et al., "Exploration of motivation to participate in a study of cancer-related cognitive impairment among patients with newly diagnosed aggressive lymphoma: a qualitative sub-study," Support Care Cancer, Sep 8 2021, doi: 10.1007/s00520-021-06527-9.

26. S. Thorne, S. Reimer Kirkham, and K. O'Flynn-Magee, "The analytic challenge in interpretive description," International Journal of Qualatative Methods, vol. 3, no. 1, pp. 1-11, 2004.

27. J. Smith and J. Firth, "Qualitative data analysis: the framework approach," Nurse Res, vol. 18, no. 2, pp. 52-62, 2011, doi: 10.7748/nr2011.01.18.2.52.c8284.

28. V. Braun and V. Clarke, "Using thematic analysis in psychology," Qualitative Research in Psychology, vol. 3, no. 2, pp. 77-101, 2008.

29. L. E. Kazis, J. J. Anderson, and R. F. Meenan, "Effect sizes for interpreting changes in health status," Med Care, vol. 27 , no. 3 Suppl, pp. S178-89, Mar 1989. [Online]. Available: https://www.ncbi.nlm.nih.gov/pubmed/2646488.

30. R Core Team R A language and environment for statistical computing. "R A language and environment for statistical computing " http://www.R-project.org/. (accessed 2019).

31. J. A. Tomas, P. A. Michael, D. Wollschaeger, and A. Omidpanah. "A Package 'epitools'." https://cran.rproject.org/web/packages/epitools/index.html (accessed.

32. S. Dorai-Raj. "Package 'binom'." https://cran.r-project.org/web/packages/binom/binom.pdf (accessed.

33. Weiss NA. "Package 'wBoot' 2016." https://cran.r-project.org/web/packages/wBoot/index.html (accessed.

34. D. Bates and M. Maechler. "Ime4 Linear Mixed-Effects Models Using Eigen and S4. ." http://CRAN.Rproject.org/package=Ime4 (accessed.

35. P. Gates, K. Gough, H. Dhillon, C. Wilson, and M. Krishnasamy, "Measurement burden in a study of cancer-related cognitive impairment. Views of patients with newly-diagnosed aggressive lymphoma. ," presented at the Multinational Association of Supportive Care in Cancer Conference, Spain, 2021.

36. D. J. Daunt, "Ethnicity and recruitment rates in clinical research studies," App/ Nurs Res, vol. 16, no. 3, pp. 189-95, Aug 2003, doi: 10.1016/s0897-1897(03)00042-9.

37. J. Lawton, The Dying Process Patients: Experiences of Palliative Care. London: Routledge, 2000.

38. E. J. C. Hay-Smith, M. Brown, L. Anderson, and G. J. Treharne, "Once a clinician, always a clinician: a systematic review to develop a typology of clinician-researcher dual-role experiences in health research with patient-participants," BMC Med Res Methodol, vol. 16, p. 95, Aug 9 2016, doi: 10.1186/s12874-016-0203-6.

39. L. Colbourne and M. Sque, "Split personalities: role conflict between the nurse and the nurse researcher," J Res Nurs, vol. 9 , no. 4, pp. 297-304, 2004.

40. M. Edwards and K. Chalmers, "Double agency in clinical research," Can J Nurs Res, vol. 34, no. 1, pp. 131-42, Jun 2002. [Online]. Available: https://www.ncbi.nlm.nih.gov/pubmed/12122770.

\section{Figures}




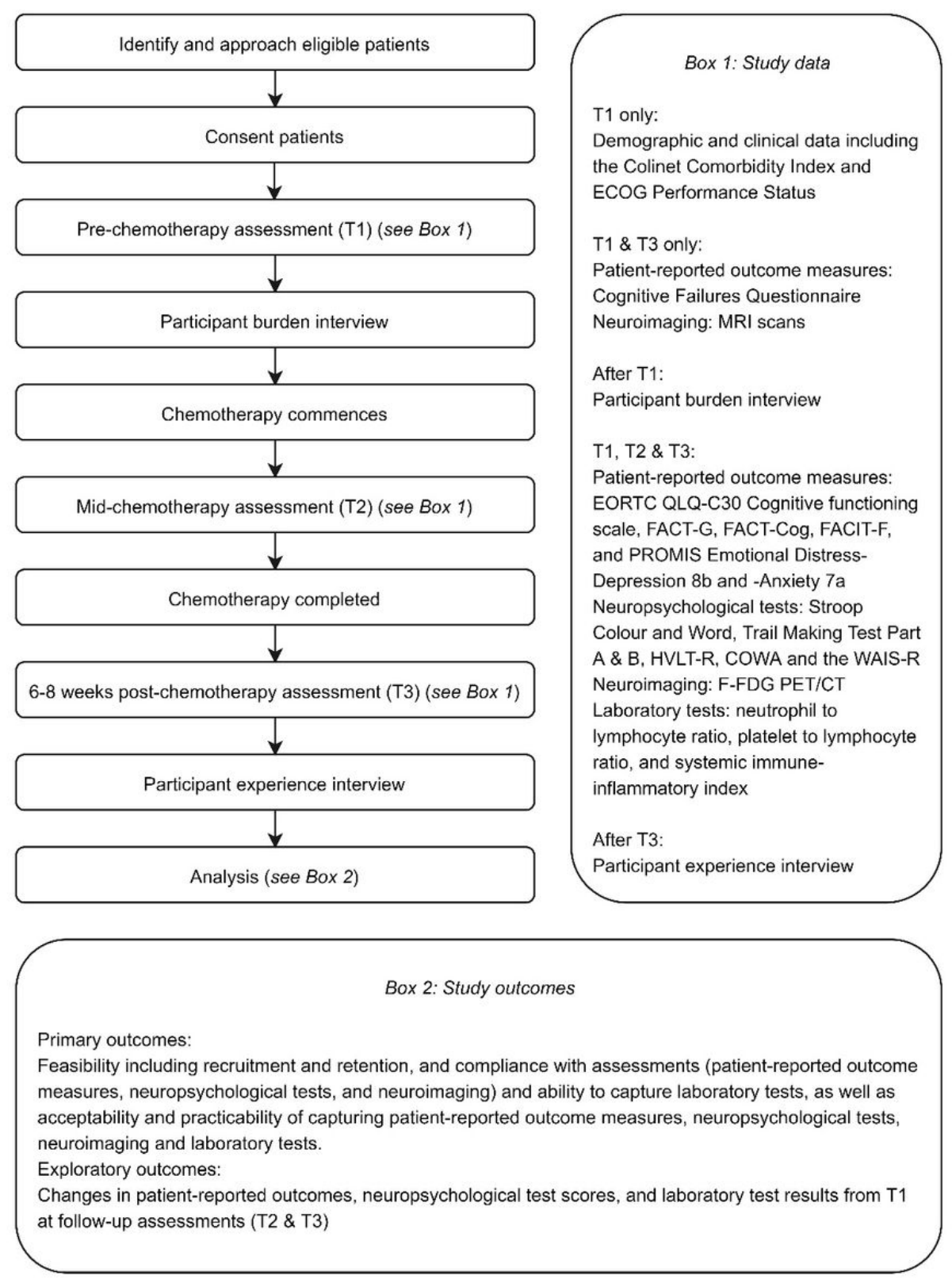

\section{Figure 1}

Study schema 


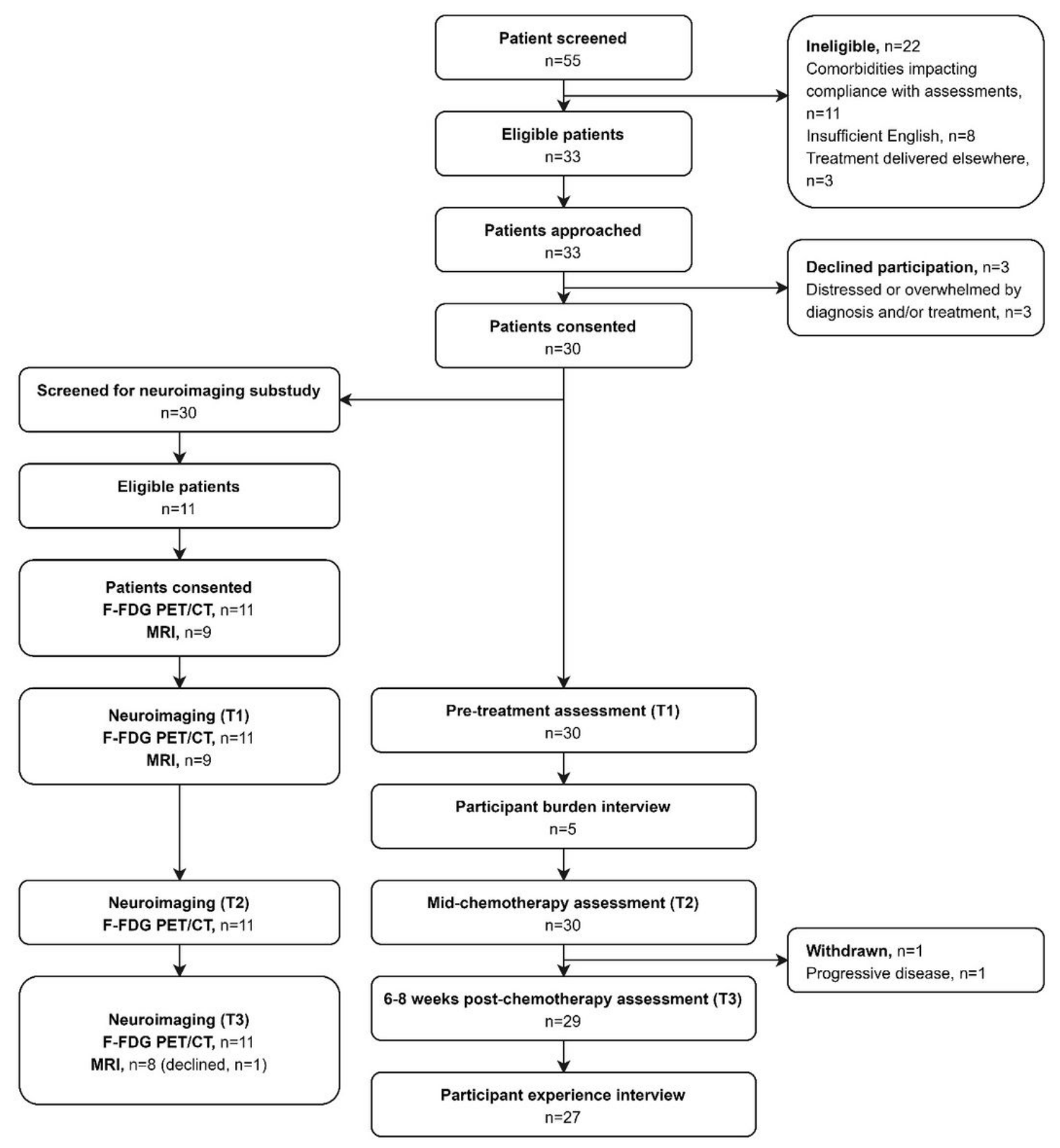

Figure 2

Participant flow diagram

\section{Supplementary Files}

This is a list of supplementary files associated with this preprint. Click to download.

- JCSSonlinesupplementalappendices24.11.21.docx 